\title{
Derivation of a Relativistic Wave Equation more Profound than Dirac's Relativistic Wave Equation
}

\author{
Koshun Suto ${ }^{1}$ \\ ${ }^{1}$ Chudaiji Buddhist Temple, Isesaki, Japan Correspondence: Koshun Suto, Chudaiji Buddhist Temple, 5-24, \\ Oote-Town, Isesaki, 372-0048, Japan \\ Correspondence: Koshun Suto, Chudaiji Buddhist Temple, Isesaki, Japan Correspondence: Koshun Suto, Chudaiji \\ Buddhist Temple, 5-24, Oote-Town, Isesaki, 372-0048, Japan. E-mail: koshun_suto129@mbr.nifty.com
}

Received: November 3, 2018

Accepted: November 22, 2018

Online Published: November 30, 2018

doi:10.5539/apr.v10n6p102

URL: https://doi.org/10.5539/apr.v10n6p102

\begin{abstract}
The author has previously derived an energy-momentum relationship applicable in a hydrogen atom. Since this relationship is taken as a departure point, there is a similarity with the Dirac's relativistic wave equation, but an equation more profound than the Dirac equation is derived. When determining the coefficients $\boldsymbol{\alpha}_{i}$ and $\beta$ of the Dirac equation, Dirac assumed that the equation satisfies the Klein-Gordon equation. The Klein-Gordon equation is an equation which quantizes Einstein's energy-momentum relationship. This paper derives an equation similar to the Klein-Gordon equation by quantizing the relationship between energy and momentum of the electron in a hydrogen atom. By looking to the Dirac equation, it is predicted that there is a relativistic wave equation which satisfies that equation, and its coefficients are determined. With the Dirac equation it is necessary to insert a term for potential energy into the equation when describing the state of the electron in a hydrogen atom. However, in this paper, a potential energy term is not introduced into the relativistic wave equation. Instead, potential energy is incorporated into the equation by changing the coefficient $\boldsymbol{\alpha}_{i}$ of the Dirac equation.
\end{abstract}

Keywords: Einstein's energy-momentum relationship, Dirac's relativistic wave equation, hydrogen atom, ultra-low energy levels, dark hydrogen, dark matter

\section{Introduction}

One of the most important relationships in the Special Theory of Relativity (STR) is as follows:

$$
\left(m_{0} c^{2}\right)^{2}+\boldsymbol{p}^{2} c^{2}=\left(m c^{2}\right)^{2} .
$$

Here, $m c^{2}$ is the relativistic energy of an object or a particle, and $m_{0} c^{2}$ is the rest mass energy.

Currently, Einstein's relationship (1) is used to describe the energy and momentum of particles in free space, but for explaining the behavior of bound electrons inside atoms, opinion has shifted to quantum mechanics as represented by equations such as the Dirac's relativistic wave equation.

For reasons such as these, there was no search for a relationship between energy and momentum applicable to an electron in a hydrogen atom.

Here, let's consider Einstein's energy-momentum relationship, that holds for isolated systems in free space. Assume now that an electron is stationary in free space. In this case, if the electron absorbs photonic energy, the photonic energy is converted to kinetic energy of the electron.

However, the situation is different if the electron is drawn toward the proton, and taken into the hydrogen atom. The kinetic energy acquired by the electron matches the emitted photonic energy. As the electron emits a photon, it also acquires kinetic energy. However, an energy source is needed to achieve this. The only conceivable possibility for this energy source is the rest mass energy of the electron.

The decrease in rest mass energy of the electron is expressed as $-\Delta m_{\mathrm{e}} c^{2}$. If the photonic energy released when an electron is drawn into a hydrogen atom is taken to be $h v$, and the kinetic energy acquired by the electron is taken to be $K$, then the following relationship holds.

$$
-\Delta m_{\mathrm{e}} c^{2}+K+h v=0
$$


In previous theory, however, the electron procured the kinetic energy and photonic energy by reducing the potential energy. The following equation expresses this situation.

$$
V(r)+K+h v=0 .
$$

The author presented the following equation as an equation indicating the relationship between the rest mass energy and potential energy of the electron in a hydrogen atom (Suto, 2009):

$$
V(r)=-\Delta m_{\mathrm{e}} c^{2} .
$$

\section{Energy-Momentum Relationship for a Bound Electron in a Hydrogen Atom}

Referring to a STR textbook (French, 1968), we derive the energy-momentum relationship for a bound electron in a hydrogen atom. When a particle moves through macroscopic space, for an isolated system, as its velocity increases, the kinetic energy and hence total mechanical energy of the particle will increase.

In classical mechanics, the increase of kinetic energy corresponds to the work done by external forces, and we have following equation.

$$
d K=F d x=\frac{d p}{d t} d x=v d p .
$$

Also, in this situation, the particle's total mechanical energy and kinetic energy increase, but the increases are equal. That is,

From Equations (5) and (6), we have,

$$
d E=d K
$$

$$
d E=v d p
$$

Next, let us imagine an electron that is at rest an infinite distance in macroscopic space from nucleus of a hydrogen atom - a proton - and is attracted by the proton's electrical force, creating a hydrogen atom. The electron emits photons outside the atom and reduces its rest mass energy, but at the same time gains an amount of kinetic energy equal to the reduced amount of energy.

The following relationship can be derived in a hydrogen atom.

$$
-d E=d K \text {. }
$$

The following relationship can be subsequently derived from Equations (5) and (8).

$$
-d E=v d p .
$$

We start from Equation (7) when deriving Einstein's relationship (1), but when deriving the relationship in a hydrogen atom, we must start from Equation (9).

In classical mechanics,

$$
m=\frac{p}{v}
$$

And, in STR,

$$
m=\frac{E}{c^{2}} .
$$

If, further, we suppose that Equation (11) describes a universal equivalence of energy and inertial mass, we can combine Equations (10) and (11) into a single statement:

$$
E=\frac{p c^{2}}{v} .
$$

Next, by multiplying the left and right sides of Equations (9) and (12), we obtain:

$$
E d E=-p c^{2} d p \text {. }
$$

We integrate this:

$$
E^{2}=-p^{2} c^{2}+\text { const }
$$

We shall next determine the constant of integration and energy $E$ for Equation (14).

The constant of integration should normally determined through experimentation. However, from the analogy of Equation (1), the constant of integration can be assumed to be $\left(m_{\mathrm{e}} c^{2}\right)^{2}$. 
Since the physical quantities of the electron in the atom have discrete values, if the subscript $n$ is attached to the physical quantities on both sides of Equation (14). Here, $n$ is the principal quantum number.

Also, the relativistic energy of the electron in a hydrogen atom is expressed as $E_{\mathrm{re}, n}$. Then, Equation (14) becomes (Suto, 2011):

$$
E_{\mathrm{re}, n}^{2}+p_{n}^{2} c^{2}=\left(m_{\mathrm{e}} c^{2}\right)^{2} .
$$

The relativistic energy of the electron $E_{\mathrm{re}, n}$ can be defined as follows.

$$
E_{\mathrm{re}, n}=m_{n} c^{2} .
$$

Here, $m_{n}$ is the mass of an electron with energy $E_{\mathrm{re}, n}$. The mass of an electron moving within an atom becomes lighter. Also, $E_{\mathrm{re}, n}$ can be defined as follows.

$$
E_{\mathrm{re}, n}=m_{\mathrm{e}} c^{2}+E_{n}=m_{\mathrm{e}} c^{2}-K_{n}=m_{\mathrm{e}} c^{2}+\frac{1}{2} V\left(r_{n}\right) .
$$

Here, $K_{n}$ is the kinetic energy of the electron.

Incidentally, Equation (12) can be written as follows.

$$
p_{n} c=\frac{E_{\mathrm{re}, n} v_{n}}{c}
$$

Here, if we substitute $p_{n} c$ in Equation (18) into Equation (15) and rearrange, then the following value is obtained.

$$
E_{\mathrm{re}, n}=m_{\mathrm{e}} c^{2}\left(1+\frac{v_{n}^{2}}{c^{2}}\right)^{-1 / 2} .
$$

\section{Elimination of Incompleteness of Equation (19)}

In its current form, Equation (19) is insufficient as a formula describing the micro world. The equation includes the velocity of the electron, and energy is not discrete.

Incidentally, Bohr's quantum condition can be written as follows.

$$
m_{\mathrm{e}} v_{n} \cdot 2 \pi r_{n}=2 \pi n \hbar
$$

Here,

$$
\hbar=\frac{h}{2 \pi}=\frac{m_{\mathrm{e}} c \lambda_{\mathrm{C}}}{2 \pi}
$$

Next, when $r_{\mathrm{e}} / \alpha$ is found,

$$
\frac{r_{\mathrm{e}}}{\alpha}=\frac{e^{2}}{4 \pi \varepsilon_{0} m_{\mathrm{e}} c^{2}} \frac{4 \pi \varepsilon_{0} \hbar c}{e^{2}}=\frac{\lambda_{\mathrm{C}}}{2 \pi}
$$

Here, $r_{\mathrm{e}}$ is the classical electron radius and $\alpha$ is the fine structure constant. Each of these can be written as follows.

$$
\begin{gathered}
r_{\mathrm{e}}=\frac{e^{2}}{4 \pi \varepsilon_{0} m_{\mathrm{e}} c^{2}}, \\
\alpha=\frac{e^{2}}{4 \pi \varepsilon_{0} \hbar c} .
\end{gathered}
$$

Also, the radius of the electron orbital derived by Bohr can be rewritten as follows.

$$
r_{n}=4 \pi \varepsilon_{0} \frac{\hbar^{2}}{m e^{2}} n^{2}=\frac{e^{2}}{4 \pi \varepsilon_{0} m_{\mathrm{e}} c^{2}}\left(\frac{4 \pi \varepsilon_{0} \hbar c}{e^{2}}\right)^{2} n^{2}=\frac{r_{\mathrm{e}}}{\alpha^{2}} n^{2}=\frac{\lambda_{\mathrm{C}}}{2 \pi \alpha} n^{2} .
$$

If the value of $r_{n}$ in Equation (25) is substituted into Equation (20), 


$$
m_{\mathrm{e}} v_{n} \frac{\lambda_{\mathrm{C}}}{\alpha} n^{2}=m_{\mathrm{e}} c \lambda_{\mathrm{C}} n
$$

Using this,

$$
\frac{v_{n}}{c}=\frac{\alpha}{n} .
$$

In a previously published paper, the author assumed Equation (27) (Suto,2014a): However, in this paper Equation (27) is not assumed. Rather, it is concluded that the left and right sides of Equation (27) are equal. It was possible to find Equation (27) from Bohr's quantum condition.

If Equation (15) is solved for energy, the following solutions can be derived.

$$
E_{\mathrm{re}, n}= \pm m_{\mathrm{e}} c^{2}\left(\frac{n^{2}}{n^{2}+\alpha^{2}}\right)^{1 / 2}, \quad n=0,1,2, \cdots
$$

Here, Equation (28) is divided into the following two equations, by taking the positive energy levels among the relativistic energy levels of the electron forming a hydrogen atom to be $E_{\mathrm{r} e, n}^{+}$, and the negative energy levels to be $E_{\mathrm{re}, n}^{-}$.

$$
\begin{aligned}
& E_{\mathrm{re}, n}^{+}=m_{\mathrm{e}} c^{2}\left(\frac{n^{2}}{n^{2}+\alpha^{2}}\right)^{1 / 2}, \quad n=1,2, \cdots \\
& E_{\mathrm{re}, n}^{-}=-m_{\mathrm{e}} c^{2}\left(\frac{n^{2}}{n^{2}+\alpha^{2}}\right)^{1 / 2}, \quad n=1,2, \cdots
\end{aligned}
$$

Incidentally, if $n=0$ then $E_{\mathrm{re}}=0$, but what can exist in this state is not the electron of a hydrogen atom. This energy is the energy of the virtual particle pairs constituting the vacuum. (This is also the energy of, respectively, the virtual electron and virtual positron.) (Suto, 2017b; Suto, 2017c): Therefore, $n=0$ was deleted in Equations (29) and (30).

When Equation (29) is used, the normal energy levels of a hydrogen atom are as follows.

$$
E_{n}=E_{\mathrm{re}, n}-m_{\mathrm{e}} c^{2}=m_{\mathrm{e}} c^{2}\left[\left(1+\frac{\alpha^{2}}{n^{2}}\right)^{-1 / 2}-1\right], \quad n=1,2, \cdots
$$

Now, if a Taylor expansion is performed on the right side of Equation (31),

$$
E_{n}=m_{\mathrm{e}} c^{2}\left[\left(1+\frac{\alpha^{2}}{n^{2}}\right)^{-1 / 2}-1\right] \approx m_{\mathrm{e}} c^{2}\left[\left(1-\frac{\alpha^{2}}{2 n^{2}}+\frac{3 \alpha^{4}}{8 n^{4}}\right)-1\right] \approx m_{\mathrm{e}} c^{2}\left(-\frac{\alpha^{2}}{2 n^{2}}+\frac{3 \alpha^{4}}{8 n^{4}}\right) .
$$

When this is done, the equations for the energies is as follows.

$$
E_{n} \approx-\frac{\alpha^{2}}{2 n^{2}} m_{\mathrm{e}} c^{2}, \quad n=1,2, \cdots
$$

Incidentally, in the classical quantum theory of Bohr, the energy levels $E_{\mathrm{B}, n}$ of a hydrogen atom are given by the following formula. (Here the B in $E_{\mathrm{B}, n}$ stands for "Bohr").

$$
E_{\mathrm{B}, n}=-\frac{1}{2}\left(\frac{1}{4 \pi \varepsilon_{0}}\right)^{2} \frac{m_{\mathrm{e}} e^{4}}{\hbar^{2}} \cdot \frac{1}{n^{2}}=-\frac{\alpha^{2}}{2 n^{2}} m_{\mathrm{e}} c^{2}, \quad n=1,2, \cdots .
$$

From this, it is evident that Bohr's energy equation, Equation (34), is an approximation of Equation (31).

The following compares energies when $n=1$.

Value predicated by this paper Equation (31): $\quad E_{1}=-13.60515 \mathrm{eV}$.

Value predicted by Bohr Equation (34): $\quad E_{\mathrm{B}, 1}=-13.60569 \mathrm{eV}$.

$$
\frac{E_{\mathrm{B}, 1}}{E_{1}}=1.0000397
$$

One could say there is almost no difference between Equations (35) and (36). However, while the energy region that can be handled with Bohr's theory is $E_{\mathrm{B}, 1} \leq E<0$, the energy region that can handled with Equation (15) is 
$-m_{\mathrm{e}} c^{2}<E_{\mathrm{re}}<m_{\mathrm{e}} c^{2}$. If Equation (15) is used, the energy region that can be handled is much broader than the region that can be handled with quantum mechanics (Suto,2018):

If a Taylor expansion is performed on the right side of Equation (30),

$$
E_{\mathrm{re}, n}^{-}=m_{n} c^{2}=-m_{\mathrm{e}} c^{2}\left(1+\frac{\alpha^{2}}{n^{2}}\right)^{-1 / 2} \approx-m_{\mathrm{e}} c^{2}+\frac{\alpha^{2}}{2 n^{2}} m_{\mathrm{e}} c^{2} .
$$

From this, we obtain,

$$
m_{n}=-m_{\mathrm{e}}\left(1-\frac{\alpha^{2}}{2 n^{2}}\right)<0
$$

Electrons at negative energy levels have negative mass. To distinguish between ordinary hydrogen atoms and this matter formed from electrons with negative mass and protons with positive mass, the author has adopted the name "dark hydrogen atom." (Suto, 2017a; Suto,2017): In this case, an aggregate of dark hydrogen atoms becomes dark hydrogen. The existence of dark hydrogen has already been discussed in another paper (Carnegie Institution for Science, 2016; McWilliams, 2016): However, the author would like to leave pending the decision on whether these two dark hydrogens are the same type.

Incidentally, the only quantum number in Equation (28) is $n$. If one wishes to obtain a solution as good as the solution of the Dirac's relativistic wave equation, an equation similar to the Dirac equation must be derived from Equation (2).

\section{Derivation of a Relativistic Wave Equation more Profound than Dirac's Relativistic Wave Equation}

The Dirac's relativistic wave equation can be written as follows (Schiff, 1968):

$$
i \hbar \frac{\partial}{\partial t} \psi=\left(-i \hbar c \alpha_{j} \nabla_{j}+\beta m_{\mathrm{e}} c^{2}\right) \psi .
$$

Here, the wave function $\psi$ is indicated by a matrix with four rows and one column.

The constants $\alpha$ and $\beta$ introduced here must satisfy relationships like the following (Dirac, 1978):

$$
\beta^{2}=1, \quad \alpha_{j} \beta+\beta \alpha_{j}=0, \quad \alpha_{j}^{2}=1, \quad \alpha_{j} \alpha_{k}+\alpha_{k} \alpha_{j}=0, \quad j, k=1,2,3(j \neq k) .
$$

It is known that the simplest matrix which satisfies these relationships is a matrix with four rows and four columns. Here, the following Pauli spin matrices and the unit matrix are used:

$$
\sigma_{1}=\left(\begin{array}{ll}
0 & 1 \\
1 & 0
\end{array}\right), \quad \sigma_{2}=\left(\begin{array}{cc}
0 & -i \\
i & 0
\end{array}\right), \quad \sigma_{3}=\left(\begin{array}{cc}
1 & 0 \\
0 & -1
\end{array}\right), \quad I=\left(\begin{array}{ll}
1 & 0 \\
0 & 1
\end{array}\right) .
$$

When this is done, the following form can be used as a matrix with four rows and four columns satisfying the condition in Equation (41):

$$
\alpha_{j}=\left(\begin{array}{ll}
0 & \sigma_{j} \\
\sigma_{j} & 0
\end{array}\right), \quad \beta=\left(\begin{array}{ll}
I & 0 \\
0 & -I
\end{array}\right) .
$$

In the era of Dirac, it was thought that Equation (1) could be applied to an electron in free space, and that the following equation incorporating potential energy into Equation (40) could be applied to an electron in an atom.

$$
i \hbar \frac{\partial}{\partial t} \psi=\left(-i \hbar c \alpha_{j} \nabla_{j}+\beta m_{\mathrm{e}} c^{2}+V\right) \psi \cdot
$$

An equation corresponding to the relationship between Equations (1) and (40) will now be derived. Now, when we perform quantization on Equation (2), we obtain the following:

$$
-\hbar^{2} \frac{\partial^{2}}{\partial t^{2}} \psi=\hbar^{2} c^{2} \nabla^{2} \psi+m_{\mathrm{e}}^{2} c^{4} \psi
$$

This equation corresponds to the Klein-Gordon equation obtained by quantizing Equation (1).

Next, the Hamiltonian describing the state of the electron in the hydrogen atom is assumed to be as follows:

$$
H=-i \hbar c \boldsymbol{\alpha}^{\prime} \cdot \nabla+\beta^{\prime} m_{\mathrm{e}} c^{2} .
$$


Here, if Equation (46) is differentiated one more time, and it is assumed that this satisfies Equation (45), then the following relationship is obtained:

$$
\left(-i \hbar c \boldsymbol{\alpha}^{\prime} \cdot \nabla+\beta^{\prime} m_{\mathrm{e}} c^{2}\right)^{2}=\hbar^{2} c^{2} \nabla^{2}+m_{\mathrm{e}}^{2} c^{4} .
$$

If this equation is written using the components of $\alpha^{\prime}$, the result is as follows:

$$
-\hbar^{2} c^{2} \sum_{j, k=1}^{3} \alpha_{j}^{\prime} \alpha_{k}^{\prime} \nabla_{j} \nabla_{k}-i m_{\mathrm{e}} c^{3} \hbar \sum_{j=1}^{3}\left(\alpha_{j}^{\prime} \beta^{\prime}+\beta^{\prime} \alpha_{j}^{\prime}\right) \nabla_{j}+\beta^{\prime 2} m_{\mathrm{e}}^{2} c^{4}=\hbar^{2} c^{2} \sum_{j=1}^{3} \nabla_{j}^{2}+m_{\mathrm{e}}^{2} c^{4}
$$

Therefore the constants $\alpha_{j}^{\prime}$ and $\beta^{\prime}$ introduced here must satisfy the following sort of relation:

$$
\beta^{2}=1, \quad \alpha_{j}^{\prime} \beta^{\prime}+\beta^{\prime} \alpha_{j}^{\prime}=0, \quad \alpha_{j}^{2}=-1, \quad \alpha_{j}^{\prime} \alpha_{k}^{\prime}+\alpha_{k}^{\prime} \alpha_{j}^{\prime}=0, \quad j, k=1,2,3(j \neq k) .
$$

The following form can be predicted for a matrix with four rows and four columns satisfying these relationships:

$$
\alpha_{j}^{\prime}=i \alpha_{j}=\left(\begin{array}{ll}
0 & i \sigma_{j} \\
i \sigma_{j} & 0
\end{array}\right), \quad \beta^{\prime}=\beta=\left(\begin{array}{ll}
I & 0 \\
0 & -I
\end{array}\right) .
$$

Using this matrix, the equation to be derived can be written as follows:

$$
\begin{aligned}
i \hbar \frac{\partial}{\partial t} \psi & =\left(-i \hbar c \alpha_{j}^{\prime} \nabla_{j}+\beta^{\prime} m_{\mathrm{e}} c^{2}\right) \psi \\
& =\left(\hbar c \alpha_{j} \nabla_{j}+\beta m_{\mathrm{e}} c^{2}\right) \psi .
\end{aligned}
$$

Incidentally, a discussion similar to this section has already been presented in another paper. However, the conclusion reached is different. In a previous paper, it was concluded that Equations (44) and (51) are on an equal footing (Suto, 2014b): However, it is not possible to derive Equation (28) from Equation (44), which takes Equation (40) as a departure point. Therefore, in this paper it is concluded that Equation (51) is superior to Equation (44) for handling the electron in a hydrogen atom.

\section{Conclusion}

In the era of Dirac, it was thought that Equation (1) could be applied to an electron in free space, and that the following equation incorporating potential energy into Equation (40) could be applied to an electron in an atom.

$$
i \hbar \frac{\partial}{\partial t} \psi=\left(-i \hbar c \alpha_{j} \nabla_{j}+\beta m_{\mathrm{e}} c^{2}+V\right) \psi .
$$

Where

$$
\alpha_{j}=\left(\begin{array}{ll}
0 & \sigma_{j} \\
\sigma_{j} & 0
\end{array}\right), \quad \beta=\left(\begin{array}{ll}
I & 0 \\
0 & -I
\end{array}\right) .
$$

In this paper, however, we have shown that the energy of a hydrogen atom can be described by the following equation as well:

$$
\begin{aligned}
i \hbar \frac{\partial}{\partial t} \psi & =\left(-i \hbar c \alpha_{j}^{\prime} \nabla_{j}+\beta^{\prime} m_{\mathrm{e}} c^{2}\right) \psi \\
& =\left(\hbar c \alpha_{j} \nabla_{j}+\beta m_{\mathrm{e}} c^{2}\right) \psi .
\end{aligned}
$$

Where

$$
\alpha_{j}^{\prime}=i \alpha_{j}=\left(\begin{array}{ll}
0 & i \sigma_{j} \\
i \sigma_{j} & 0
\end{array}\right), \quad \beta^{\prime}=\beta=\left(\begin{array}{ll}
I & 0 \\
0 & -I
\end{array}\right) .
$$

Equation (52) was obtained by inserting a term $V$ into the hamiltonian in Equation (40). On the other hand, Equation (54) was obtained by changing the coefficient in Equation (40) from $\boldsymbol{\alpha}_{j}(53)$ to $\boldsymbol{\alpha}_{j}^{\prime}(55)$. The change in 
this coefficient corresponds to incorporating potential energy into the hamiltonian in Equation (40). Because the Dirac equation (40) does not include potential energy, the term $V$ has been newly added in Equation (44). However, $V$ is already included within $E_{\mathrm{re}, n}$ in Equation (2).

The problem is solved not by adding $V$ to the Dirac equation (40), but by changing the coefficient from $\boldsymbol{\alpha}_{j}$ to $\boldsymbol{\alpha}_{j}^{\prime}$.

Incidentally, Equation (2) has a solution that cannot be predicted with the Dirac equation. Therefore, this paper concludes that, when treating the problem of the hydrogen atom, Equation (54) derived from Equation (2) is superior to the Dirac equation (52).

\section{Acknowledgements}

I would like to express my thanks to the staff at ACN Translation Services for their translation assistance.

\section{References}

Carnegie Institution for Science. (2016). Probing giant planets' dark hydrogen, June 23.

Dirac, P. A. M. (1978). Directions in Physics (p. 56). New York: Wiley.

French, A. P. (1968). Special Relativity (p. 21). New York \& London: W.W.NORTON \&COMPANY.

McWilliams, R. S., Dalton, D. A., Mahmood, M. F., \& Goncharov, A. F. (2016). Optical properties of fluid hydrogen at the transition to a conducting state. Physical Review Letters, 116, 255501. https://doi.org/10.1103/PhysRevLett.116.255501

Schiff, L. I. (1968). Quantum Mechanics (p. 472). New York: McGraw-Hill.

Suto, K. (20017a). Presentation of dark matter candidates, Applied Physics Research, 9(1), 70-76. http://dx.doi.org/10.5539/apr.v9n1p70

Suto, K. (2009). True nature of potential energy of a hydrogen atom. Physics Essays, 22(2), 135-139. http://dx.doi.org/10.4006/1.3092779

Suto, K. (2011). An energy-momentum relationship for a bound electron inside a hydrogen atom. Physics Essays, 24(2), 301-307. http://dx.doi.org/10.4006/1.3583810

Suto, K. (2014a). $n=0$ energy level present in the hydrogen atom. Applied Physics Research, 6(5), 109-115. http://dx.doi.org/10.5539/apr.v6n5p109

Suto, K. (2014b). Previously unknown ultra-low energy level of the hydrogen atom whose existence can be predicted, Applied Physics Research, 6(6), 64-73. http://dx.doi.org/10.5539/apr.v6n6p64

Suto, K. (2017). Region of dark matter present in the hydrogen atom, Journal of Physical Mathematics, 8, 4. https://doi.org/10.4172/2090-0902.1000252

Suto, K. (2017b). Elucidating the nature of the fine structure constant and indicating the existence of an unknown an gular momentum, Applied Physics Research, 9(3), 17-22. https://doi.org/10.5539/apr.v9n3p17

Suto, K. (2017c). Previously unknown physical formulas which hold in a hydrogen atom and are derived without using quantum mechanics, Applied Physics Research, 9(4), 7-16. https://doi.org/10.5539/apr.v9n4p7

Suto, K. (2018). Potential energy of the electron in a hydrogen atom and a model of a virtual particle pair constituting the vacuum, Applied Physics Research, 10(4), 93-101. https://doi.org/10.5539/apr.v10n4p93

\section{Copyrights}

Copyright for this article is retained by the author(s), with first publication rights granted to the journal.

This is an open-access article distributed under the terms and conditions of the Creative Commons Attribution license (http://creativecommons.org/licenses/by/4.0/). 